\title{
Image Segmentation using MSNCut Algorithm
}

\author{
Basavaprasad B. \\ Bharathiar University \\ Coimbatore - 641046 \\ India
}

\begin{abstract}
In this paper a hybrid technique for the segmentation of image has been proposed and it is named as MSNCut technique. Here image segmentation is the process of dividing the given image into number of regions that possess similar properties such as color, texture and intensity which are useful for the image analysis. The image is generic here, in other words image may be tree, river, building, medical or any general image. In this proposed method first the input image is preprocessed by mean shift algorithm to divide it into its constituent regions. Then the resultant image is represented as a bi-partite graph and finally the resultant graph (image) is processed under normalized cut to classify the image into meaningful classes.
\end{abstract}

\section{General Terms}

Image Segmentation, Algorithm, Performance, Space Complexity, Pixels, Hybrid et. al.

\section{Keywords}

Segmentation, MSNCut, Mean Shift, Minimal cut, Clustering.

\section{INTRODUCTION}

The main motto of image segmentation is to analyses the image which is an important phase in image processing. The image may be generic or medical. Image segmentation helps to develop the applications such as OCR (Optical Character Recognition), satellite image classification, remote sensing, face recognition, traffic signal location, medical image segmentation and many other. During the last few decades' image segmentation turned out to be one of major tool in medical area used for the further analysis.

Image segmentation is a process in which the image is mainly divided into two sub-regions referred as foreground and background. Here foreground is the required area of analysis in other words output. And the background is the unwanted area in other words the region that is not used in image analysis in image processing. Further image processing can be divided into three stages which are shown in Fig. 1. First level is the low-level in which image is operated at the pixel-level. The second level is middle level in which the image is analyzed after it is being segmented. Finally, in the third level called as high level the image is understood for further processing such as classification, feature extraction etc.

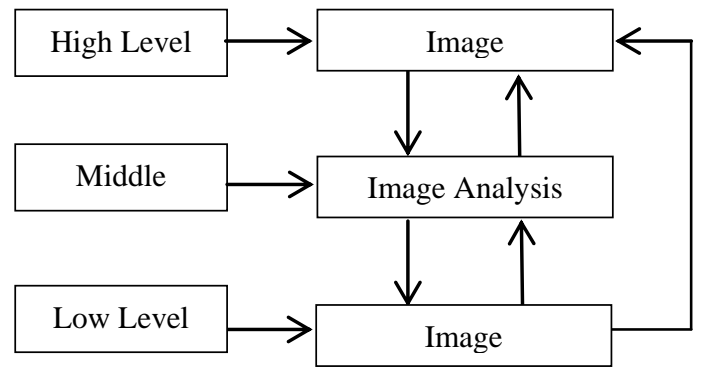

Figure 1. Image Segmentation Process for image classification
The proposed work is hybrid which combines the mean shift algorithm and normalized cut algorithm to perform faster and in a more efficient manner. During the section 2 explanation of the related work has been presented. Section 3 contains the proposed our work. Section 4 consists of results and discussions. Finally, in section 5 details of references is mentioned.

\section{LITERATURE SURVEY}

There are plenty of image segmentation algorithms available which are used in image analysis but graph based image segmentation algorithms are more efficient and cost effective. These algorithms are more effective when combined with other techniques such as statistical, fuzzy based and with traditional methods also.

\subsection{Image Clustering using Mean Shift Algorithm}

Mean shift algorithm is a clustering algorithm which functions in feature space. In this paper the transitory analysis of image segmentation technique based on mean shift algorithm [4] has been presented. It involves two stages. During first stage, the input image is improved into feature space using a nonparametric technique i.e., it is converted into kernel density to assess the model features. And in the second stage, the image segmentation problem can be deliberated as a clustering problem by determining the methods of density function and allocating point to the modes. For instance, for defining kernel density approximation at point $x$ in a $\operatorname{dataset}\left\{x_{i}\right\}_{i=1}^{n} \subseteq R^{D}$.

$f^{\prime}=\frac{1}{n} \sum_{i=1}^{n} K_{H}\left(x-x_{i}\right)$

where $D$ is the dimension of the data. For easiness, the kernel widths as the same have assumed. Now the kernel density estimation becomes:

$f^{\prime}(x)=\frac{1}{n} \sum_{i=1}^{n} k\left(\left\|\frac{x-x_{i}}{h}\right\|\right)^{2}$

The mean shift vector is given by:

$\mathrm{m}(\mathrm{x})=\frac{\sum_{\mathrm{i}=1}^{\mathrm{n}} \mathrm{x}_{\mathrm{i}} \mathrm{k}\left(\left\|\frac{\mathrm{x}-\mathrm{x}_{\mathrm{i}}}{\mathrm{h}}\right\|\right)^{2}}{\sum_{\mathrm{i}=1}^{\mathrm{n}} \mathrm{k}\left(\left\|\frac{\mathrm{x}-\mathrm{x}_{\mathrm{i}}}{\mathrm{h}}\right\|\right)^{2}}-\mathrm{x}$

Now iteration of $x^{T+1}=m\left(x^{T}\right)$ has been done .For every point, the structure congregates to a mode of the density function (3). Later for each point which congregates to the same mode, a cluster has been allocated. Consequently, the final segmented image has been obtained.

\subsection{Spectral Graph Partitioning}

In this paper, one of the above methods that is Normalized cut (Ncut) for our experimentation is used. The image is denoted by a graph with $G=(V, E, W)$, where $V$ represents the collection of nodes and $E$ corresponds to the collection of edges which connects the nodes. The nodes $u$ and $v$ are connected using an edge e and is weighted by $w(u, v)=$ $\mathrm{w}(\mathrm{v}, \mathrm{u}) \geq 0$ which measures the dissimilarity amongst the nodes. $W$ is an edge similarity matrix with $w(u, v)$ as its $(\mathrm{u}, \mathrm{v})$ the element [1]. The graph which represents an image is subdivided into two separate sub-graphs $A$ and $B=V-A$ 
just by eliminating that edge which connect nodes in $A$ with nodes in $B$. This is called bipartite-graph. Then the degree of dissimilarity amid these two sets into a total weight of the eliminated edges using the equation is calculated,

$\operatorname{cut}(A, B)=\sum_{u \in A, v \in B} w(u, v)$

During the minimum cut algorithm the cut value has been minimized. Nevertheless, minimum cut constantly tries to cut small collection of isolated nodes as the cut which is shown in (3) does not contain any information of intragroup [7].

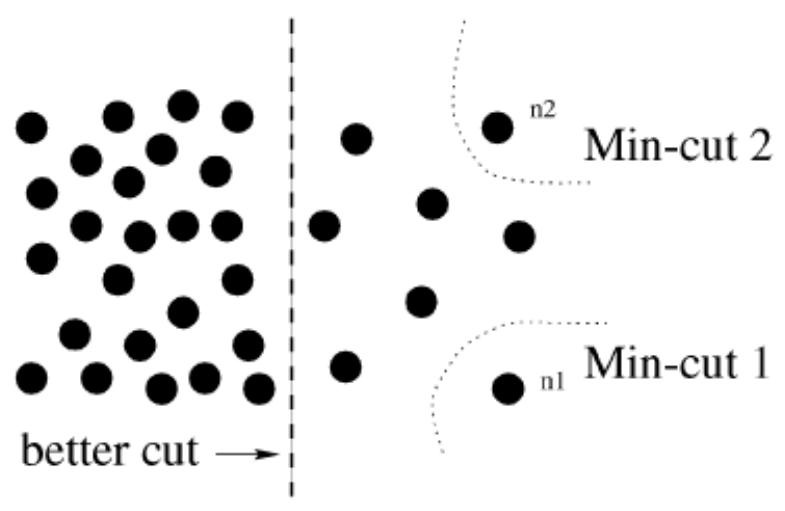

Fig.2. The minimum cut which results in bad partition [7].

Because of this the normalized cut algorithm is used to solve this problem, by using the formula:

$\operatorname{Ncut}(A, B)=\frac{\operatorname{cut}(A, B)}{\operatorname{assoc}(A, V)}+\frac{\operatorname{cut}(A, B)}{\operatorname{assoc}(B, V)}$

Here $\operatorname{assoc}(A, V)$ shows the connection between the nodes in $A$ with all nodes in the given graph, and $\operatorname{assoc}(B, V)$ is the similarly for the sub-graph $B$. Therefore, by reducing the disassociation among the groups and maximizing the connotation within groups is obtained at the same time.

\section{PROPOSED APPROACH}

\subsection{Description of algorithm}

In this section the proposed hybrid algorithm is explained. The summary scheme of proposed algorithm can be regarded as the following. To begin with, an image is sub-divided into several disjoint regions. Mean shift algorithm [4] has been used to achieve this. Next, the graph of clustered regions is constructed which represents each cluster has one node and the value of dissimilarity among the clusters is defined. To finish, a graph partitioning algorithm using normalized cut technique [1] is used to get both the final segmentation and classification at the same time.

Lot of survey has been done on image segmentation. Based on this, the problem is framed in a way that the segments formed by mean shift algorithm can be represented by a planar weighted graph which is called as region adjacency graph (RAG) $G=(V, E, W)$ which integrate the narrative information of the structure of image and connectivity of segments. Therefore, the graph containing clusters represented by the RAG algorithm and link neighboring regions by an edge. The value of dissimilarity $W$ between neighborhood regions defined by defining the color space. The weight matrix $W$ can be calculated for all regions. The weight $\mathrm{w}(\mathrm{u}, \mathrm{v})$ between regions $u$ and $v$ is defined as

$w(w, v)=\left\{\begin{array}{cc}e^{-\left[\frac{\|\mathrm{F}(\mathrm{u})-\mathrm{F}(\mathrm{v})\|_{2}^{2}}{\mathrm{~d}_{\mathrm{I}}}\right]} & \text { If } \mathrm{u} \text { and } \mathrm{v} \text { are adjacent } \\ 0, & \text { otherwise }\end{array}\right.$
Where $\mathrm{F}(\mathrm{u})=\{\mathrm{L}(\mathrm{u}), \mathrm{u}(\mathrm{u}), \mathrm{v}(\mathrm{u})\}$ defines the color vector for region $u$ and the symbol $\|.\|_{2}$ represents the vector standard operator. $d_{I}$ is a positive measuring factor for defining the compassion of $\mathrm{w}(\mathrm{u}, \mathrm{v})$ to the color variation between nodes $u$ and $v$. By this graph demonstration of the image, region assembling can be formulated as a graph-partitioning problem (called as bi-partite graph). For solving this problem Ncut algorithm is used [1]. But our method has a slight difference with those because it segments and classify the image parallel. The explanation is given about this in the following section.

\subsection{An innovative method for classification image}

This section gives the details of classification of an image using the proposed method in which the concept of based on graph based technique has been reused for segmenting the image. The procedure of algorithm is explained in above segment. But the important quota of our method that delivers a technique for image classification based on bi-partite graph i.e., partitioning in the graph during building stage. Throughout the previous approach, each two neighborhood nodes which represents regions connected by an edge based on RAG algorithm. However, in this technique all the nodes are collected connected. In other words there is no such neighboring constraint to calculate the measure of dissimilarity between the two pixel values $W$ for every edge between nodes.

$\mathrm{w}(\mathrm{w}, \mathrm{v})=\mathrm{e}^{-\left[\frac{\|\mathrm{F}(\mathrm{u})-\mathrm{F}(\mathrm{v})\|_{2}^{2}}{\mathrm{~d}_{\mathrm{I}}}\right]}$

The overall work can be summarized through the algorithm which is named as MSncut is as follows.

\section{Algorithm MSNcut}

Step 1: Apply mean shift to pixel representations

quite tightly clustered, numerous, local minima is obtained colour balancing, space and position space inversely alters results

Step 2: Apply k-means to local minima

numerous segments are to be segmented

but have a tendency to be much better clustered than pixel images

pixel fit in to segment whose number is equal to the number of local centre

Step 3: Graph structure partitioning (dividing the image into regions or clusters)

Step 4: Apply normalized cut to segment the input image to get the required output segments.

\section{Advantages of mean shift algorithm}

The algorithm is very simple to implement.

The complete end result is measured by one and only one parameter which is the value of kernel bandwidth, whereas further clustering approaches, for example, k-means, needs the input of a number of clusters to be stated.

Mean shift smartly feats the density of the points in an effort to produce a sensible number of clusters.

The kernel bandwidth value can regular times are chosen grounded on specific domain-specific information. 


\section{Disadvantages of mean shift algorithm}

The most obvious disadvantage is its lateness, more precisely; it is an $\mathrm{N}$ squared algorithm. For the problems which are having many points, it takes a long time to execute.

\section{Advantages of normalized cut}

Presence of an impartial measure, the Ncut value corresponding to the isolated nodes will be of a big percentage equated to the total connection from slight set to all other nodes unlike in graph cuts.

The computation time of normalised cut is $0(\mathrm{mn})$.Where $m$ the number of steps is and $n$ is the number of pixels in the given input image.

\section{Disadvantage of normalised cut}

One disadvantage of normalised cut is the computational time. It takes more computational time compared to the many hybrid methods of image segmentation.

\section{Advantage of the proposed method}

The hybridised proposed method authorizes normalised cut algorithms to execute segmentation on image independently i.e. part by part, rather than accomplishing segmentation by implementing mean shift algorithm only.

Segmentation results are clearer with respect to visual perception.

\section{RESULTS AND DISCUSSIONS}

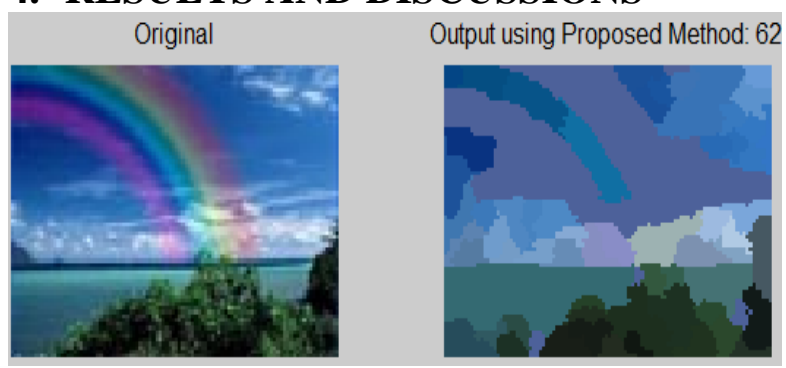

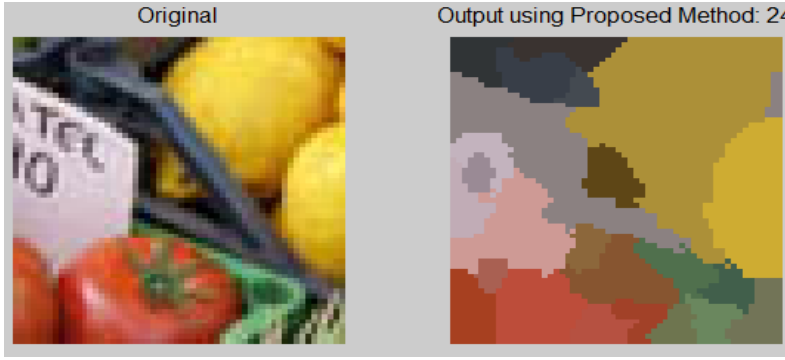
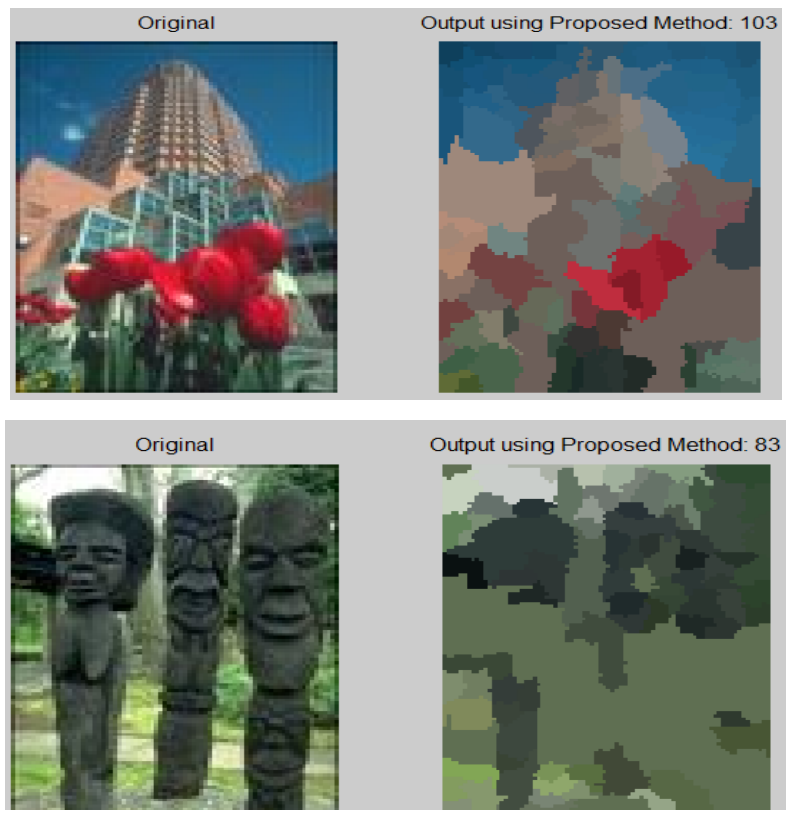

Figure 1. Image Segmentation Process for image classification

Discrimination is much clearer in proposed method compared with other graph cut methods. A hybrid method combining normalized cuts algorithm with mean shift algorithm in a further well-organized way has been experimented for the segmentation of images. The standard database is used i.e. Bakerly Dataset for our experimentation and have tested over 200 generic images. Matlab 2014 is used for programming purpose with system having higher or latest configuration.

The same results we have showed in this section.

Table 1. Comparison of various graph based algorithms with the proposed method.

\begin{tabular}{|l|l|l|l|l|l|}
\hline & $\begin{array}{l}\text { Min-Cut/Max- } \\
\text { Flow }\end{array}$ & Random Walker & MST-Based & Normalized Cut & Proposed Method \\
\hline Applications & $\begin{array}{l}\text { Photo/video } \\
\text { editing, } \\
\text { Psychological } \\
\text { vision and } \\
\text { medical image } \\
\text { segmentation }\end{array}$ & $\begin{array}{l}\text { object/background } \\
\text { segmentation and } \\
\text { Medical image } \\
\text { segmentation }\end{array}$ & $\begin{array}{l}\text { Generic image } \\
\text { segmentation } \\
\text { Object } \\
\text { segmentation and }\end{array}$ & $\begin{array}{l}\text { Natural scene } \\
\text { image } \\
\text { segmentation }\end{array}$ & $\begin{array}{l}\text { Natural scene } \\
\text { image } \\
\text { segmentation, } \\
\text { psychological } \\
\text { vision and } \\
\text { medical image } \\
\text { segmentation }\end{array}$ \\
\hline $\begin{array}{l}\text { Testing } \\
\text { Dataset }\end{array}$ & Bakerly Dataset & Bakerly Dataset & Bakerly Dataset & Bakerly Dataset & Bakerly Dataset \\
\hline
\end{tabular}




\begin{tabular}{|l|l|l|l|l|l|}
\hline Performance & $\begin{array}{l}\text { Less than a } \\
\text { second for most } \\
\text { 2D images (up } \\
\text { to 512*512) }\end{array}$ & $\begin{array}{l}\text { Around 3 seconds on a } \\
256 * 256 \text { image for an } \\
\text { Intel Xeon 2.40GHz } \\
\text { with 3GB RAM }\end{array}$ & None & $\begin{array}{l}2 \text { mins on Intel } \\
\text { Pentium } \\
\text { 200MHz } \\
\text { machines for } \\
100 * 120 \text { test } \\
\text { image }\end{array}$ & $\begin{array}{l}\text { 0.5863 seconds } \\
\text { are required on a } \\
1.4 \mathrm{GHz} \text { AMD } \\
\text { Athlon with 512k } \\
\text { RAM }\end{array}$ \\
\hline $\begin{array}{l}\text { Time } \\
\text { Complexity }\end{array}$ & $\begin{array}{l}O\left(m n^{2}\right) \text { in worst } \\
\text { case, } n \text { is the } \\
\text { number of } \\
\text { nodes and } m \text { is } \\
\text { the number of } \\
\text { edges }\end{array}$ & $\begin{array}{l}O(n), n \text { is the number of } \\
\text { nodes in the graph }\end{array}$ & $\begin{array}{l}O(m \log m) \text { for } m \\
\text { graph edges }\end{array}$ & $\begin{array}{l}O(m n), n \text { is the } \\
\text { number of pixels } \\
\text { and } m \text { is the } \\
\text { number of steps } \\
\text { for convergence }\end{array}$ & $\begin{array}{l}O(m+n \log n), n \text { is } \\
\text { the number of } \\
\text { nodes and } m \text { is the } \\
\text { number of edges }\end{array}$ \\
\hline
\end{tabular}

\section{CONCLUSIONS}

In the proposed work, a hybrid algorithm been constructed which has been named as MSNCut is grounded on the view that a solution for segmentation of general images is achieved. By treating the work as a hybrid method which is the combination of mean shift algorithm a graph partitioning problem and normalized cut the required results have been achieved. A computational technique grounded on this knowledge has been developed and applied to segmentation of color, brightness and texture images. The results of the proposed experiments on general images are very encouraging and demonstrate that he MSNcut criterion does definitely satisfy our original objective of segmenting the image.

\section{ACKNOWLEDGMENTS}

My thanks to my guide Dr. Ravindra S Hegadi who have contributed towards development of the paper work. I also thank hearty to the Research and Development Center, Bharathiar University, Coimbatore-641046 for giving me the opportunity to do research work.

\section{REFERENCES}

[1] Malik J and Shi J, "Normalized Cuts and Image Segmentation", IEEE transactions on pattern analysis and machine intelligence, vol. 22, no. 8, 2000.

[2] Nida M. Zaitoun, Musbah J and Aqel, "Survey on Image Segmentation Techniques", International Conference on Communication, Management and Information Technology (ICCMIT), 2015.
[3] Nameirakpam D, Khumanthem M and Yambem J. C, "Image Segmentation using K-means Clustering Algorithm and Subtractive Clustering Algorithm", Eleventh International Multi-Conference on Information Processing, 2015.

[4] Cheng and Yizong, "Mean Shift, Mode Seeking and Clustering"', An IEEE Transaction on Pattern Analysis and Machine Intelligence, Volume: 17, Issue:8, pp:790799, 1995.

[5] Basavaprasad B and Ravi M, "Enhanced Color Image Segmentation by Graph Cut Method in General and Medical Image", Lecture Notes in Bioengineering, Advancements of Medical Electronics, Proceedings of the First International Conference, ICAME, 2015.

[6] Shi J and Malik J, "Image Segmentation and the Normalized Cuts", Machine Intelligence and Pattern Analysis, an IEEE Transactions, Volume 22, 2000.

[7] Basavaprasad B, Ravindra S Hegadi, "Color Image Segmentation Using Adaptive GrowCut Method", International Conference on Advanced Computing Technologies and Applications (ICACTA), 2015

[8] Fulkerson D and Ford L, "Flow in networks", Princeton University Press, 1962.

[9] Koschan Aand Skarbek W, "A Survey on Color Image Segmentation", Technical Report, Tech. University of Berlin, 1994. 